\title{
Effects of active acromegaly on bone mRNA and microRNA expression patterns
}

\section{Zhanna Belaya', Tatiana Grebennikova', Galina Melnichenko', Alexey Nikitin², Alexander Solodovnikov ${ }^{3}$, Olga Brovkina², Andrey Grigoriev', Liudmila Rozhinskaya', Alexander Lutsenko ${ }^{1}$ and Ivan Dedov ${ }^{1}$}

${ }^{1}$ The National Medical Research Centre for Endocrinology, Moscow, Russia, ${ }^{2}$ Federal Research and Clinical Center FMBA of Russia, Moscow, Russia, and ${ }^{3}$ Ural State Medical Academy, Ekaterinburg, Russia
Correspondence should be addressed to Z Belaya Email jannabelaya@gmail.com

\begin{abstract}
Objective: To evaluate the response of bone to chronic long-term growth hormone (GH) and insulin-like growth factor-1 (IGF1) excess by measuring the expression of selected mRNA and microRNA (miR) in bone tissue samples of patients with active acromegaly.

Design: Case-control study.

Methods: Bone tissue samples were obtained during transsphenoidal adenomectomy from the sphenoid bone (sella turcica) from 14 patients with clinically and biochemically confirmed acromegaly and 10 patients with clinically nonfunctioning pituitary adenoma (NFPA) matched by sex and age. Expression of genes involved in the regulation of bone remodeling was studied using quantitative polymerase chain reaction (qPCR).

Results: Of the genes involved in osteoblast and osteoclast activity, only alkaline phosphatase (ALP) mRNA was $50 \%$ downregulated in patients with acromegaly. GH excess caused increased expression of the Wnt signaling antagonists (DKK1) and agonists (WNT1OB) and changes in the levels of miR involved in mesenchymal stem cell commitment to chondrocytes (miR-199a-5p) or adipocytes (miR-27-5p, miR-125b-5p, miR-34a-5p, miR-188-3p) $P<0.05 ; q<0.1$. Relevant compensatory mechanisms were found through the changes in miR involved in osteoblastogenesis (miR-210-5p, miR135a-5p, miR-211, miR-23a-3p, miR-204-5p), but the expression of TWIST1 was 50\% downregulated and RUNX2 was unchanged.

Conclusions: Acromegaly had minimal effects on tested mRNAs specific to osteoblast or osteoclast function except for downregulated ALP expression. The expressions of miR known to be involved in mesenchymal stem cell commitment and downregulated TWIST1 expression suggest acromegaly has a negative effect on osteoblastogenesis.
\end{abstract}

\section{Introduction}

Growth hormone (GH) and insulin-like growth factor-1 (IGF1) are important regulators of bone remodeling in children and adults $(1,2)$. In vitro $\mathrm{GH}$ stimulates the proliferation, differentiation and function of osteoblasts (1).

Acromegaly is caused by GH-producing pituitary adenoma and results in chronically elevated GH and IGF1 concentration (3). Several studies suggested that
C) 2018 European Society of Endocrinology Printed in Great Britain acromegaly has protective effects on bone mineral density (BMD) $(2,4)$ and even decreases fracture risk (5). However, a recent meta-analysis of forty-one studies showed that while acromegalic patients had unaltered lumbar spine BMD and higher femoral neck BMD, an increased risk of vertebral fracture was seen (6). This increased fracture risk may be explained by the altered quality of bone rather than $\operatorname{BMD}$ loss $(7,8)$. 
Interestingly, biochemical markers of bone formation and bone resorption were both reported to be increased in patients with acromegaly as compared to control subjects, but markers of bone resorption tended to be more increased in relation to bone formation (6). Similar changes in bone turnover markers are reported in patients with hyperparathyroidism, thyrotoxicosis and postmenopausal osteoporosis and are usually associated with markedly decreased BMD (9), contrary to the unchanged or even increased BMD in subjects with acromegaly $(2,4,6)$. The molecular mechanisms of these alterations in humans are generally unknown.

In this study, we examined the effects of chronic GH and IGF1 excess on the expression of mRNA and microRNA (miR) known to affect bone homeostasis in bone tissue samples from patients with active acromegaly.

\section{Subjects and methods}

The Institutional Review Board of the National Research Centre for Endocrinology (NRCE) approved the study protocol.

Fourteen patients with clinically evident and biochemically proven acromegaly and ten patients with benign clinically non-functioning pituitary adenoma (NFPA), who had signed a formal informed consent form, were enrolled into the study.

Acromegaly patients had typical clinical manifestations, including acral and facial changes, an elevated IGF1 (normalized for age and sex) and lack of suppression of $\mathrm{GH}$ to $<1 \mathrm{ng} / \mathrm{mL}$ following documented hyperglycemia during an oral glucose load (3). All subjects underwent magnetic resonance imaging (MRI) where the presence of a pituitary adenoma was confirmed. Among patients with acromegaly, amenorrhea was diagnosed in four cases, all other women reported normal menstrual function. One patient had type 2 diabetes with appropriately controlled glucose on $500 \mathrm{mg}$ metformin therapy. All patients were treatment naïve with regards to somatostatin analogues or cabergoline (as mentioned in the exclusion criteria). Four patients with acromegaly received ACE inhibitors to control blood pressure.

The patients with NFPA had pituitary hormone levels within the reference range (10) and were referred to surgery because of optic chiasm compression in nine cases and in one case because of severe headache. Two patients from the NFPA group regularly used ACE inhibitors to treat hypertension.
The groups were not different regarding menstrual function and testosterone levels. Three patients from the acromegaly group and three patients from the NFPA group reported smoking. Their levels of physical activity were normal - none were athletic, immobile or had physically demanding job. None of the enrolled patients took calcium supplements, but all of them consumed dairy products in their diet. The enrollment period was from October to May.

Exclusion criteria were pregnancy, glucocorticoid use, alcohol abuse, exacerbation of chronic disease, severe conditions (i.e., renal and liver insufficiency, heart attack, stroke), terminal conditions, prolonged immobilization ( $>1$ week), clinically evident fracture within the previous six months, any other cause of secondary osteoporosis within the past five years or any prolonged treatment with drugs documented to influence bone metabolism in humans during the previous 12 months (11) including treatment with antiresorptive or anabolic compounds for osteoporosis or treatment with a somatostatin analogue.

\section{Serum measurements}

All subjects had fasting 08:00-09:00 h serum samples taken to obtain routine biochemistry, hormonal status and bone turnover markers (octeocalcin (OC), carboxyterminal cross-linked telopeptide of type I collagen (CTx)).

Serum samples of OC, CTx, TSH, prolactin, ACTH, cortisol in serum and saliva were assayed by electrochemiluminescence (ECLIA) Cobas 6000 Module e601 Roche. Vitamin D $\left(25 \mathrm{OHD}_{3}\right)$, IGF1 and GH levels were measured by immunochemiluminescence assay (Liaison). Routine biochemistry was performed on Architect 8000 (Abbott).

\section{Bone mineral density and fracture assessment}

At the time of enrollment all participants were questioned regarding any recent low traumatic fractures, back pain and height changes. Height was measured by stadiometer and a body mass index (BMI) was calculated as kilograms per meter squared.

Patients underwent standard spinal radiographs in anterior-posterior and lateral positions of the vertebrae Th4-L4 (Axiom Icons R200 'Siemens'). A deformity was considered a fracture if the visual inspection perceived a reduction in vertebral height (anterior, posterior or middle) of $20 \%$ or more (12).

BMD was measured by dual-energy X-ray absorptiometry (iDXA, GE) at the anteroposterior lumbar 
spine (L1-L4) and femoral neck (the lower of two $Z$-scores was chosen) positions according to standard protocol. Quality control procedures were carried out in accordance with the manufacturer's recommendations.

\section{Sample collection and RNA isolation}

Bone tissue samples were taken during a transsphenoidal adenomectomy from the sphenoid bone (base of the sella turcica) before the removal of the pituitary adenoma and were washed with sterile $0.9 \% \mathrm{NaCl}$ solution to remove visible blood. Bone tissue samples were trimmed using a scalpel to a uniform size of $5 \times 5 \mathrm{~mm}$ and were immediately placed in lysis buffer ( $1 \mathrm{~mL}$ QIAzol Lysis Reagent; Qiagen, 79306) and homogenized by a TissueLyser LT. Total RNA isolation from bone tissue with on-column digestion of the genomic DNA was carried out with a miR Neasy Mini Kit (Qiagen, 217004) on the automatic 'QIAcube' station as per the manufacturer's protocol. To prevent degradation 1 unit of RiboLock RNase Inhibitor (Thermo Fisher Scientific, EO0382) was added per $1 \mu \mathrm{L}$ of RNA solution. The concentration of total RNA in the aqueous solution was evaluated on a NanoVue Plus spectrophotometer (GE Healthcare).

\section{Quantification of messenger RNA (mRNA) transcripts by qRT-PCR}

A two-step quantitative reverse transcriptase-polymerase chain reaction (qRT-PCR) was carried out using a HighCapacity RNA-to-cDNA Kit (Thermo Fisher, 4368814) and Custom TaqMan Array 48 Plus plates (Thermo Fisher Scientific, 4413258), run in 96-well format on the StepOnePlus instrument (Applied Biosystems), according to the manufacturer's protocol. Data analyses were done using SDS Software (version 2.3, Applied Biosystems). All samples were normalized to GUSB and levels of GAPDH, HPRT served as secondary internal controls.

\section{Quantification of microRNA (miRNA, miR) transcripts by qRT-PCR}

A qRT-PCR was carried out using a TaqMan Advanced miRNA cDNA Synthesis Kit (Thermo Fisher, A28007) and TaqMan Advanced miRNA Assays (Thermo Fisher Scientific, A25576), run in 96-well format on the StepOnePlus instrument (Applied Biosystems), according to the manufacturer's protocol. All samples were normalized to hsa-miR-191 levels and spike-ink control
cel-miR-39-3p. Data analyses were performed using SDS Software (version 2.3, Applied Biosystems).

\section{Statistical analysis}

Data are presented using mean (M) values and 95\% confidence intervals $(95 \% \mathrm{CI})$. Comparisons between the descriptive parameters of patients with acromegaly and NFPA were made using non-paired, 2-sided $t$-tests. Fisher's exact test was used to compare two independent groups for qualitative parameters.

All statistical analysis and graphical output were performed using R software, version 3.4.0 (2017-04-21). The original scale and log2-transformed data were plotted using a 'ggplot' package and descriptive statistics were calculated using a basic 'stats' package. Each parameter was tested using a paired $t$-test to evaluate any differences between acromegaly and NFPA. Spearman's rank test was used to correlate quantitative parameters within the groups.

A $P$ value less than 0.05 was considered statistically significant. In order to account for multiple comparisons, a correction for the false discovery rate $(q$ values $<0.10)$ was calculated using the Benjamini-Hochberg adjustment $(13,14)$.

No power calculations were made for this study in advance, and there was no statistical hypothesis as it was an exploratory rather than confirmatory study. Yet, post hoc power analysis was conducted on the samples, and it was confirmed that for the majority of comparisons made the power of the calculation exceeded 90\% (ranging from 39.2\% to $99.9 \%$ across mRNA and miRNA parameters tested).

\section{Results}

The general characteristics and biochemical parameters of participants are summarized in Table 1. Subjects with acromegaly had elevated levels of IGF1 - 792 (95\%CI: 582-1003) ng/mL and basal GH - 10.1 (2.34-22.5) ng/ $\mathrm{mL}$ with the mean estimated duration of acromegaly being approximately 7 (3-10) years. Subjects with NFPA had IGF1 - 110 (45-175) ng/mL and all other pituitary hormone levels within the reference range. Although patients with NFPA tended to be older (not statistically significant $P=0.056)$, bone remodeling markers were higher in patients with acromegaly $(P<0.05)$, making age a non-significant factor. More patients with acromegaly (three vs one) had low traumatic fractures, but the difference was not statistically significant (Table 1). 
Table 1 General characteristics of patients with acromegaly and clinically non-functioning pituitary adenoma (NFPA).

\begin{tabular}{l} 
Variable \\
\hline$n$ \\
Age (years) \\
Body mass index $\left(\mathrm{kg} / \mathrm{m}^{2}\right)$ \\
Sex female $(\%): \mathrm{male}(\%)$ \\
Calcium $(\mathrm{mmol} / \mathrm{L})$ \\
Creatinine $(\mathrm{mkmol} / \mathrm{L})$ \\
Osteocalcin $(\mathrm{ng} / \mathrm{mL})$ \\
Carboxyterminal cross-linked telopeptide \\
$\quad$ of type I collagen $(\mathrm{ng} / \mathrm{mL})$ \\
Vitamin D $(\mathrm{ng} / \mathrm{mL})$ \\
L1-L4 (g/cm²) \\
L1-L4 Z-score \\
Femur neck (g/cm²) \\
Femur neck Z-score \\
Low traumatic fractures $N(\%)$ \\
Type of Fracture
\end{tabular}

\begin{tabular}{c}
\hline Acromegaly mean $(95 \% \mathrm{Cl})$ \\
\hline 14 \\
$37(32-41)$ \\
$28(25-31)$ \\
$8(51.8): 6(48.2)$ \\
$2.38(2.32-2.44)$ \\
$65(59-71)$ \\
$48.6(30.7-66.5)$ \\
$1.16(0.67-1.64)$ \\
$10.9(8.4-13.6)$ \\
$1.029(0.801-1.258)$ \\
$0.2(-0.5$ to 1.1$)$ \\
$0.964(0.824-1.104)$ \\
$0.6(-0.3$ to 1.43$)$ \\
$3(21 \%)$
\end{tabular}

In 2 cases vertebral fractures and in 1 case non-vertebral fracture (radius)

\begin{tabular}{cccc}
\hline NFPA mean $(95 \% \mathrm{Cl})$ & & $\boldsymbol{P}$ value \\
\cline { 1 - 1 } 10 & & \\
$45(33-57)$ & & 0.057 \\
$27(23-31)$ & & 0.941 \\
$6(60): 4(40)$ & & 0.895 \\
$2.35(2.33-2.43)$ & & 0.742 \\
$72(64-79)$ & & 0.116 \\
$23.5(12.3-34.6)$ & & 0.015 \\
$0.58(0.28-0.89)$ & & 0.039 \\
& & \\
$12.2(5.9-18.4)$ & & 0.879 \\
$1.039(0.879-1.199)$ & & 0.392 \\
$-0.4(-1.8$ to 0.9$)$ & & 0.302 \\
$0.865(0.723-1.007)$ & & 0.375 \\
$-0.2(-1.2$ to 0.7$)$ & & 0.167 \\
$1(10 \%)$ & & 0.468
\end{tabular}

In 1 case vertebral fracture

\section{Effects of acromegaly on gene expression critical for osteoblast differentiation and maturation}

As shown in Fig. 1 and Table 2, no significant changes in the expressions of genes responsible for osteoblast function and bone formation $(15,16,17)$ were seen apart from alkaline phosphatase $(A L P)$, which was $50 \%$ downregulated in subjects with acromegaly vs NFPA. The expression of some growth factors that affect osteoblastogenesis (17) (BMP2, IGF1, VEGFA) were downregulated in bone tissue samples of acromegaly patients, but BMP7 was upregulated. TWIST1, but not RUNX2, was almost 50\% downregulated. Among the key extracellular regulators of the Wnt signaling pathway $(15,16)$, we found a 10 -fold upregulation of WNT1OB $(P=0.001 ; q=0.017)$ and increased expression of the Wnt signaling antagonist DKK1 (7.7 (4.7-10.7) $P<0.001$; $q=0.011)$. The expression of SOST was upregulated $(P=0.043)$, but this was not significant when the correction on multiple comparisons was applied $(q=1)$ (Table 2).

We did not find any difference in the expressions of SFRP1 or WNT3A.

We performed correlation analysis between IGF1, basal GH and expression of all tested genes (Table 2) in both groups of patients together. We found significant correlation between IGF1 levels and the following gene expressions: ALP: $\rho_{\mathrm{o}}=-0.579, P=0.012 ; B M P 7: \rho_{\mathrm{o}}=0.683$, $P=0.002 ; D K K 1: \rho_{\mathrm{o}}=0.618, P=0.006 ;$ FGFR1: $\rho_{\mathrm{o}}=0.706$, $P=0.001 ;$ HPRT1: $\rho_{\mathrm{o}}=-0.689, P=0.002 ;$ IGF1: $\rho_{\mathrm{o}}=-0.642$, $P=0.004 ;$ IGFBP2: $\rho_{\mathrm{o}}=-0.469, P=0.050 ;$ IL6: $\rho_{\mathrm{o}}=-0.768$, $P<0.001$; IL6R: $\rho_{\mathrm{o}}=-0.577, P=0.012$; leptin: $\rho_{\mathrm{o}}=-0.802$, $P=0.003 ; \quad P C D H A 6: \quad \rho_{0}=-0.765, \quad P<0.001 ; \quad S F R P 1$ : $\rho_{\mathrm{o}}=-0.529, P=0.024 ;$ SOST: $\rho_{\mathrm{o}}=0.818, P<0.001 ;$ STAT1: $\rho_{\mathrm{o}}=0.500, \quad P=0.035 ; \quad$ TWIST1: $\rho_{\mathrm{o}}=-0.810, \quad P<0.001$; VEGFA: $\rho_{\mathrm{o}}=-0.803, \quad P<0.001 ; \quad W N T 10 B: \quad \rho_{\mathrm{o}}=0.701$, $P=0.003$. The only significant correlation found between basal GH and mRNA levels was SOST: $\rho_{\mathrm{o}}=0.812, P=0.008$. However, basal GH showed significant positive correlation with serum levels of osteocalcin: $\rho_{\mathrm{o}}=0.821, P=0.023$ and CTx: $\rho_{\mathrm{o}}=0.810, P=0.015$. The relation between IGF1 and CTx was less evident $\rho_{\mathrm{o}}=0.636 P=0.011$ and did not reach a statistically significant value when we tested the IGF1 correlation with osteocalcin $\rho_{\mathrm{o}}=0.503 P=0.067$.

Neither IGF1, nor GH levels showed any significant correlation with COL1A1, COL1A2 or BGLAP gene expression in bone tissue samples.

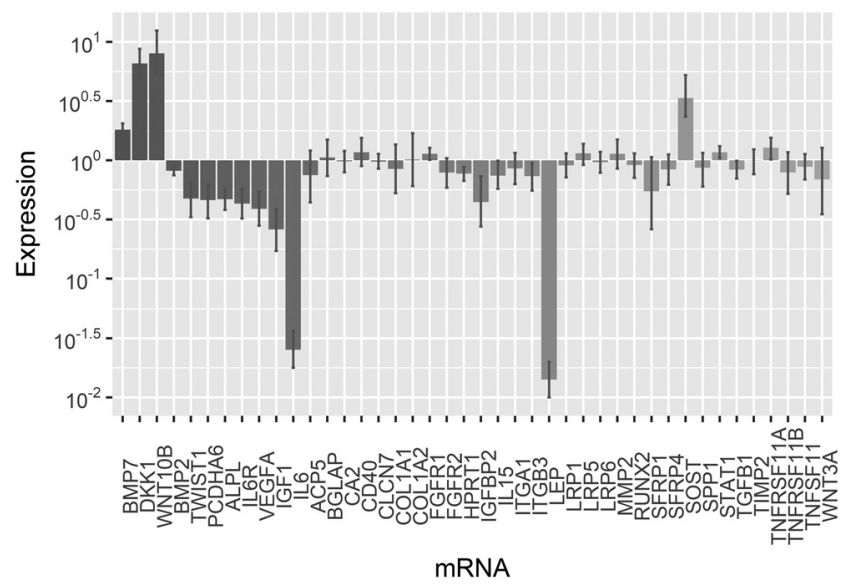

Figure 1

The effects of growth hormone excess on relative mRNA levels in bone tissue samples of patients with active acromegaly. 
Table 2 mRNA levels in bone samples of patients with acromegaly compared with clinically non-functioning pituitary adenoma (NFPA) patients.

\begin{tabular}{|c|}
\hline Gene symbol \\
\hline$A C P 5$ \\
\hline$A L P$ \\
\hline$B G L A P$ \\
\hline$B M P 2$ \\
\hline$B M P 7$ \\
\hline CA2 \\
\hline$C D 40$ \\
\hline CLCN7 \\
\hline COL1A1 \\
\hline COL1A2 \\
\hline$D K K 1$ \\
\hline FGFR1 \\
\hline FGFR2 \\
\hline HPRT1 \\
\hline IGF1 \\
\hline IGFBP2 \\
\hline IL15 \\
\hline IL6 \\
\hline IL6R \\
\hline ITGA1 \\
\hline ITGB3 \\
\hline$L R P 1$ \\
\hline LRP5 \\
\hline LRP6 \\
\hline MMP2 \\
\hline PCDHA6 \\
\hline$R U N X 2$ \\
\hline SFRP1 \\
\hline SFRP4 \\
\hline SOST \\
\hline SPP1 \\
\hline STAT1 \\
\hline TGFB1 \\
\hline TIMP2 \\
\hline TNFRSF11A (RANK) \\
\hline TNFRSF11B (OPG) \\
\hline TNFSF11 (RANKL) \\
\hline TWIST1 \\
\hline VEGFA \\
\hline WNT10B \\
\hline WNT3A \\
\hline
\end{tabular}

\begin{tabular}{l}
\hline Gene name \\
\hline Acid phosphatase 5 tartrate resistant \\
Alkaline phosphatase \\
Bone gamma-carboxyglutamate protein \\
Bone morphogenetic protein 2 \\
Bone morphogenetic protein 7 \\
Carbonic anhydrase 2 \\
CD 40 molecule
\end{tabular}

N

Fibroblast growth factor receptor 2

Hypoxanthine phosphoribosyltransferase 1

Insulin-like growth factor 1

Insulin-like growth factor binding protein 2

Interleukin 15

Interleukin 6

Interleukin 6 receptor

Integrin subunit alpha 1

Integrin subunit beta 3

Low-density lipoprotein receptor protein 1

Low-density lipoprotein receptor protein 5

Low-density lipoprotein receptor protein 6

Matrix metallopeptidase 2

Protocadherin alpha 6

Runt related transcription factor 2

Secreted frizzled related protein 1

Secreted frizzled related protein 4

Sclerostin

Secreted phosphoprotein 1

Signal transducer and activator of transcription 1

Transforming growth factor beta-1

TIMP metallopeptidase inhibitor 2

TNF receptor superfamily member 11 a (Receptor

Activator of Nuclear Factor $\kappa$ B)

TNF receptor superfamily member $11 b$ (osteoprotegerin)

TNF superfamily member 11 (Receptor activator of nuclear factor kappa-B ligand)

Twist family bHLH transcription factor 1

Vascular endothelial growth factor A

Wnt family member $10 \mathrm{~B}$

Wnt family member $3 \mathrm{~A}$

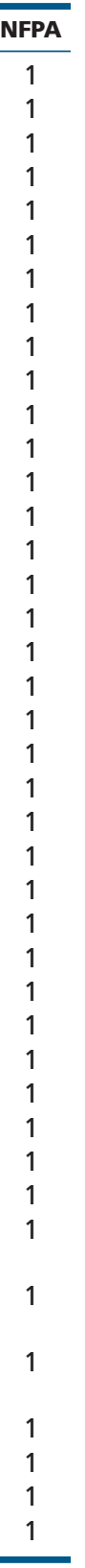

\begin{tabular}{c}
\hline Acromegaly mean $(95 \% \mathrm{CI})$ \\
\hline $1.09(0.59-1.59)$ \\
$0.51(0.39-0.62)$ \\
$1.36(0.74-1.97)$ \\
$0.82(0.75-0.90)$ \\
$1.86(1.59-2.14)$ \\
$1.05(0.82-1.29)$ \\
$1.33(0.94-1.71)$ \\
$1.01(0.83-1.19)$ \\
$1.24(0.57-1.92)$ \\
$1.69(0.61-2.76)$ \\
$7.7(4.69-10.71)$ \\
$1.16(1.02-1.31)$ \\
$0.89(0.66-1.12)$ \\
$0.8(0.67-0.94)$ \\
$0.33(0.20-0.47)$ \\
$0.67(0.24-1.11)$ \\
$0.85(0.58-1.12)$ \\
$0.03(0.02-0.05)$ \\
$0.49(0.36-0.63)$ \\
$0.99(0.69-1.28)$ \\
$0.82(0.60-1.04)$ \\
$0.99(0.77-1.20)$ \\
$1.24(0.98-1.49)$ \\
$1.04(0.81-1.27)$ \\
$1.3(0.87-1.74)$ \\
$0.55(0.38-0.72)$ \\
$1.01(0.76-1.25)$ \\
$1(0.38-1.62)$ \\
$0.97(0.68-1.27)$ \\
$5.18(1.16-9.20)$ \\
$1.02(0.72-1.31)$ \\
$1.2(1.04-1.35)$ \\
$0.88(0.71-1.04)$ \\
$1.08(0.86-1.30)$ \\
$1.38(1.06-1.69)$ \\
$1.05(0.51-1.60)$ \\
$0.98(0.74-1.22)$ \\
$0.56(0.41-0.71)$ \\
$0.48(0.30-0.65)$ \\
$10.11(5.96-14.26)$ \\
$1.18(0.41-1.96)$ \\
\hline
\end{tabular}

\begin{tabular}{rlll}
\hline \multicolumn{1}{c}{$\boldsymbol{P}$} & & $\boldsymbol{Q}$ \\
\cline { 1 - 1 } 0.704 & & 1 \\
$<0.001$ & & $<0.001$ \\
0.235 & & 1 & \\
$<0.001$ & & 0.011 \\
$<0.001$ & & 0.001
\end{tabular}

0.629

0.093

0.918

0.45

0.192

$<0.001$

0.032

0.327

0.007

$<0.001$

0.131

0.256

$<0.001$

$<0.001$

0.922

0.095

0.887

0.066

0.705

0.156

$<0.001$

0.965

0.998

0.851

0.043

0.91

0.019

0.136

0.445

0.022

1

1

1

1

1

0.011

0.858

1

0.217

$<0.001$

1

1

$<0.001$

$<0.001$

1

1

1

1

1

0.003

1

1

1

1

1

1

0.607

$0.831 \quad 1$

$0.849 \quad 1$

$<0.001 \quad 0.001$

$<0.001 \quad 0.001$

$0.001 \quad 0.017$

0.619

1

Analyzing the association between serum bone remodeling markers and gene expressions patterns, we found significant correlation between osteocalcin and the following mRNA levels: $D K K 1: \rho_{\mathrm{o}}=0.562, P=0.015$; FGFR1: $\rho_{\mathrm{o}}=0.667, P=0.002$; HPRT1: $\rho_{\mathrm{o}}=-0.515, P=0.029$; SOST: $\rho_{\mathrm{o}}=0.732, P=0.001$; TNFSF11: $\rho_{\mathrm{o}}=0.486, P=0.041$. We also found association between CTx and FGFR1: $\rho_{\mathrm{o}}=0.664, P=0.002 ;$ SOST: $\rho_{\mathrm{o}}=0.607, P=0.006$; TNFSF11: $\rho_{\mathrm{o}}=0.484, P=0.041$.
Neither OC, nor CTx levels demonstrated any correlations with COL1A1, COL1A2, BGLAP or ALP gene expression in bone tissue samples.

Of the tested miRs (Fig. 2 and Table 3), we found significant downregulation of miR-29a-3p, miR-29b-3p and miR-29c-3p, which may have negative effects on Wnt signaling $(18,19)$ and therefore negatively affect osteoblastogenesis. At the same time, we also found upregulation of miR-125b-5p, miR-188-3p and miR- 


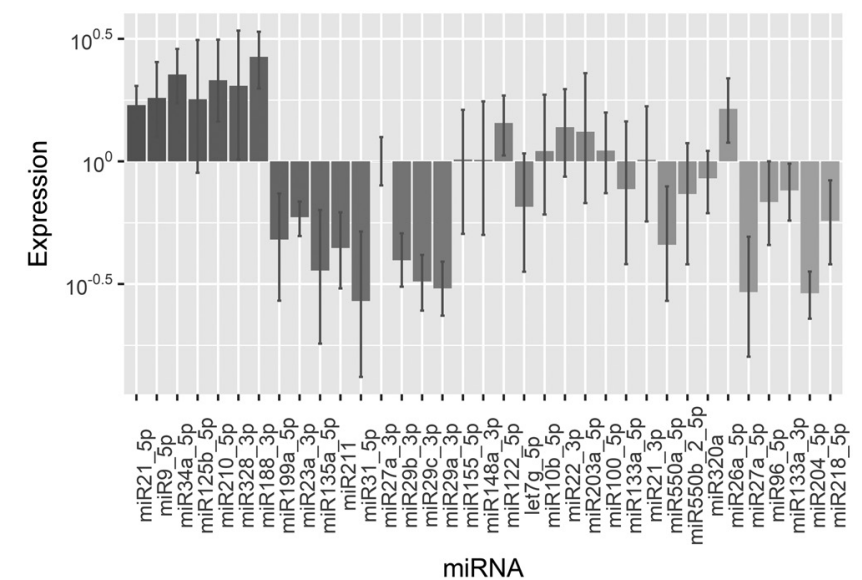

Figure 2

The effects of growth hormone excess on relative microRNA (miR) levels in bone tissue samples of patients with active acromegaly.

$34 a-5 p$, which are expected to have negative effects on osteoblastogenesis and positive effects on adipogenesis $(20,21,22,23)$. Similar negative effects on osteogenic differentiation $(24,25,26)$ might be expected from miR-27a-5p downregulation (Table 3). The observed downregulation of miR-199a-5p might be beneficial for mesenchymal stem cell commitment to chondrocytes over osteoblasts (27).

In contrast to this, upregulation of miR-210-5p (Fig. 2 and Table 3) should promote osteoblastogenesis by targeting its negative regulator (28). Furthermore, several miRs which functionally inhibit the differentiation of osteoprogenitors by attenuating RUNX2 and SMAD5 pathways (miR-135a-5p; miR-211; mirR-204-5p; miR$23 a-3 p)(29,30,31)$ were downregulated in bone tissue samples of patients with acromegaly.

The expression of several more miR with less defined functions, but also involved in bone remodeling $(32,33)$ were changed. miR-9-5p and miR-328-3p were upregulated more than twofold. The expression of miR-21-5p was $80 \%$ increased and miR-31-5p was 50\% downregulated (Fig. 2 and Table 3).

Correlation analysis showed significant associations between serum IGF1 and the following miR: miR-135a-5p: $\rho_{\mathrm{O}}=-0.528, P=0.024 ; \mathrm{miR}-125 \mathrm{~b}-5 \mathrm{p}: \rho_{\mathrm{o}}=0.610, P=0.007$; miR-211: $\rho_{\mathrm{o}}=-0.530, P=0.029 ; \operatorname{miR}-31-5 \mathrm{p}: \rho_{\mathrm{o}}=-0.737$, $P<0.001$; miR-34a-5p: $\rho_{\mathrm{o}}=0.691, P=0.001$; miR-550a-5p: $\rho_{\mathrm{o}}=-0.554, P=0.017 ; \mathrm{miR}-27 \mathrm{a}-5 \mathrm{p}: \rho_{\mathrm{o}}=-0.613, P=0.007$; miR-188-3p: $\rho_{\mathrm{o}}=0.65,2 P=0.003$; miR-133a-3p: $\rho_{\mathrm{o}}=-0.652$, $P=0.003 ; \quad \operatorname{miR}-204-5 \mathrm{p}: \quad \rho_{\mathrm{o}}=-0.940, \quad P<0.001 ; \quad \mathrm{miR}-$ 23a-3p: $\rho_{\mathrm{o}}=-0.584, \quad P=0.011 ; \operatorname{miR}-27 \mathrm{a}-5 \mathrm{p}: \rho_{\mathrm{o}}=-0.613$,
$P=0.007$; miR-29a-3p: $\rho_{\mathrm{o}}=-0.698, P=0.001 ; \mathrm{miR}-29 \mathrm{~b}-3 \mathrm{p}$ : $\rho_{\mathrm{o}}=-0.510, P=0.03$; miR-29c-3p: $\rho_{\mathrm{o}}=-0.633, P=0.005$.

Basal GH levels were associated with miR-31-5p: $\rho_{\mathrm{o}}=-0.733, \quad P=0.025 ; \mathrm{miR}-188-3 \mathrm{p}: \rho_{\mathrm{o}}=0.917, \quad P=0.001$; miR-204-5p: $\quad \rho_{\mathrm{o}}=-0.845, \quad P=0.004$ and miR-22-3p: $\rho_{\mathrm{o}}=0.700, P=0.036$ among all enrolled patients.

Regarding bone remodeling markers, we found the following association between osteocalcin and miRs: miR-21-5p: $\rho_{\mathrm{o}}=0.482, \quad P=0.043 ;$ miR-31-5p: $\rho_{\mathrm{o}}=-0.487$, $P=0.041$; miR-34a-5p: $\rho_{\mathrm{o}}=0.520, P=0.027$; miR-27a-5p: $\rho_{\mathrm{o}}=-0.632, P=0.005 ; \mathrm{miR}-188-3 \mathrm{p}: \rho_{\mathrm{o}}=0.585, \quad P=0.011$; miR-204-5p: $\rho_{\mathrm{o}}=-0.679, P=0.002$. Some correlations were also found between serum CTx levels and miR- 125b5p: $\rho_{\mathrm{o}}=0.519, P=0.023$; miR-34a-5p: $\rho_{\mathrm{o}}=0.457, P=0.049$; miR-27a-5p: $\rho_{\mathrm{o}}=-0.632, P=0.026$; miR-188-3p: $\rho_{\mathrm{o}}=0.506$, $P=0.027$; miR-204-5p: $\rho_{\mathrm{o}}=-0.678, P=0.001$.

\section{Effects of acromegaly on gene expressions critical for osteoclast activation and function}

The mRNA levels of genes responsible for osteoclast function and osteoclastogenesis (34) were mostly unchanged, including the RANKL/RANK/OPG pathway. PCDHA6 (responsible for adhesion and cell to cell interaction (35)), IL-6 and its receptor were downregulated (Table 2).

We did not observe significant correlation between serum vitamin D (25OHD3) levels and mRNA or miR expressions. We did not find any statistically significant correlations between IGF1 and Vitamin D or GH and vitamin D levels.

We did not find any difference in mRNA or microRNA levels in subjects who had previously sustained low traumatic fractures. Still the number of patients with fractures was small and the fractures were not clinically evident at the moment of enrollment.

\section{Discussion}

This is the first study evaluating both mRNA and microRNA expression profiles in bone tissue samples of patients with active acromegaly. We found significantly increased expression of the Wnt signaling antagonist (DKK1) and agonist (WNT1OB) and changes in microRNA expressions involved in mesenchymal stem cell commitment to enhance chondrocyte and adipocyte over osteoblast development.

Consistent with previous reports (6), increased bone formation and bone resorption markers were found in 
Table 3 miRNA levels in bone samples of patients with active acromegaly compared with clinically non-functioning pituitary adenoma (NFPA) patients.

\begin{tabular}{l}
\hline MicroRNA (miR) symbol \\
\hline miR21-5p \\
miR210-5p \\
miR135a-5p \\
miR155-5p \\
miR148a-3p \\
miR122-5p \\
miR125b-5p \\
miR9-5p \\
miR328-3p \\
miR211 \\
miR31-5p \\
miR34a-5p \\
let7b-5p \\
miR10b-5p \\
miR22-3p \\
miR203a-5p \\
miR100-5p \\
miR133a-5p \\
miR21-3p \\
miR550a-5p \\
miR550b-2-5p \\
miR199a-5p \\
miR320a \\
miR26a-5p \\
miR27a-5p \\
miR96-5p \\
miR188-3p \\
miR133a-3p \\
miR204-5p \\
miR218-5p \\
miR23a-3p \\
miR27a-3p \\
miR29a-3p \\
miR29b-3p \\
miR29c-3p \\
\hline
\end{tabular}

\begin{tabular}{|c|}
\hline NFPA \\
\hline 1 \\
\hline 1 \\
\hline 1 \\
\hline 1 \\
\hline 1 \\
\hline 1 \\
\hline 1 \\
\hline 1 \\
\hline 1 \\
\hline 1 \\
\hline 1 \\
\hline 1 \\
\hline 1 \\
\hline 1 \\
\hline 1 \\
\hline 1 \\
\hline 1 \\
\hline 1 \\
\hline 1 \\
\hline 1 \\
\hline 1 \\
\hline 1 \\
\hline 1 \\
\hline 1 \\
\hline 1 \\
\hline 1 \\
\hline 1 \\
\hline 1 \\
\hline 1 \\
\hline 1 \\
\hline 1 \\
\hline 1 \\
\hline 1 \\
\hline 1 \\
\hline 1 \\
\hline
\end{tabular}

\begin{tabular}{c}
\hline Acromegaly mean (95\% confidence interval) \\
\hline $1.8(1.45-2.15)$ \\
$2.66(1.85-3.48)$ \\
$0.55(0.36-0.75)$ \\
$1.38(0.93-1.83)$ \\
$1.51(0.97-2.05)$ \\
$1.64(1.18-2.10)$ \\
$2.62(1.75-3.49)$ \\
$2.23(1.48-2.98)$ \\
$2.91(2.03-3.79)$ \\
$0.53(0.38-0.68)$ \\
$0.45(0.25-0.65)$ \\
$2.52(1.87-3.16)$ \\
$0.96(0.57-1.35)$ \\
$1.64(1.01-2.27)$ \\
$1.72(1.21-2.23)$ \\
$2.12(1.21-3.03)$ \\
$1.38(0.91-1.85)$ \\
$1.34(0.71-1.97)$ \\
$1.48(0.92-2.05)$ \\
$0.69(0.40-0.99)$ \\
$1.03(0.68-1.38)$ \\
$0.63(0.45-0.82)$ \\
$0.97(0.72-1.22)$ \\
$1.94(1.29-2.58)$ \\
$0.44(0.25-0.62)$ \\
$0.86(0.56-1.16)$ \\
$2.95(2.33-3.56)$ \\
$0.86(0.62-1.09)$ \\
$0.31(0.25-0.38)$ \\
$0.75(0.45-1.04)$ \\
$0.62(0.52-0.73)$ \\
$1.08(0.82-1.34)$ \\
$0.34(0.24-0.44)$ \\
$0.44(0.30-0.58)$ \\
$0.36(0.27-0.45)$ \\
\end{tabular}

\begin{tabular}{c}
\hline $\boldsymbol{P}$ \\
\hline$<0.001$ \\
0.001 \\
$<0.001$ \\
0.092 \\
0.063 \\
0.01 \\
0.001 \\
0.004 \\
$<0.001$ \\
$<0.001$ \\
$<0.001$ \\
$<0.001$ \\
0.84 \\
0.048 \\
0.009 \\
0.02 \\
0.104 \\
0.265 \\
0.087 \\
0.043 \\
0.865 \\
0.001 \\
0.805 \\
0.008 \\
$<0.001$ \\
0.332 \\
$<0.001$ \\
0.207 \\
$<0.001$ \\
0.089 \\
$<0.001$ \\
0.502 \\
$<0.001$ \\
$<0.001$ \\
$<0.001$ \\
\hline
\end{tabular}

\begin{tabular}{c}
\hline \multicolumn{1}{c}{$\boldsymbol{Q}$} \\
\hline 0.007 \\
0.017 \\
0.007 \\
0.959 \\
0.757 \\
0.156 \\
0.03 \\
0.07 \\
0.01 \\
$<0.001$ \\
0.001 \\
0.006 \\
1 \\
0.621 \\
0.153 \\
0.298 \\
0.959 \\
1 \\
0.959 \\
0.604 \\
1 \\
0.022 \\
1 \\
0.138 \\
0.001 \\
1 \\
$<0.001$ \\
1 \\
$<0.001$ \\
0.959 \\
$<0.001$ \\
1 \\
$<0.001$ \\
$<0.001$ \\
$<0.001$ \\
\end{tabular}

serum samples of patients with acromegaly. However, we did not find significant upregulation of tested genes responsible for osteoblast or osteoclast function. Rather unexpectedly, $A L P$, which is a marker of mature osteoblast activity, was 50\% downregulated along with TWIST1. The data were supported by strong negative correlations between serum IGF1 and both ALP and TWIST1 expression. Consequently, the increased bone remodeling markers in serum samples of patients with acromegaly cannot be explained by the increase in bone remodeling rate. Contrary to this, analysis of gene expression patterns in bone tissue samples of postmenopausal women (10 postmenopausal vs 7 premenopausal) (36) showed upregulated expressions of all tested collagen molecules, BGLAP, ALP, RUNX2, TNFSF11/RANKL and many other genes which clearly demonstrate an increased bone remodeling rate. This explains the increased bone remodeling markers in serum samples in postmenopausal women (9).

Thus, the previously reported changes in gene expressions patterns in bone samples of postmenopausal women (36) differ from the currently reported in patients with acromegaly, though bone remodeling markers are elevated in both conditions $(6,9)$.

In an adult skeleton endochondral ossification occurs during the fracture healing process, which is also characterized by markedly increased bone remodeling markers in serum (37). Nevertheless, none of the enrolled patients with acromegaly had had a fracture within the previous six months, but all had obvious visible signs of acromegaly, which indicates active cartilage metabolism. Consequently, we suggest that cartilage growth in acromegaly may explain the increased bone remodeling markers in serum, contrary to the increased 
bone remodeling rate usually observed in other forms of secondary osteoporosis or postmenopausal osteoporosis (9). This hypothesis was also supported by the absence of correlations between OC or CTx in serum and COL1A1, COL1A2, BGLAP or ALP gene expression in bone tissue samples.

During normal endochondral ossification, ALP secretion is vital for mineral deposition and the formation of osteoprogenitor cells that secrete osteoid to form the bone trabecula (37). Theoretically, a fifty percent downregulation of ALP in bone of patients with acromegaly may alter the later stages of bone matrix production (trabecula formation, osteoblast maturation) and therefore it may explain the altered quality of newly formed bone.

Expression of IGF1 in bone tissue samples was downregulated, most likely due to the reciprocal changes in response to high serum IGF1 levels. $B M P 7$, upregulated in the present study, induces the phosphorylation of SMAD1 and SMAD5, that are important transcription factors for osteogenic differentiation of mesenchymal stem cells (38). With regards to the Wnt signaling components, both the Wnt signaling agonist (WNT1OB) and antagonist (DKK1) were upregulated, but the key osteoblast transcription factor (RUNX2) was unchanged. This is probably due to an achieved balance in the tight regulation of bone remodeling in long-term acromegaly.

In addition to mRNA, we analyzed miR expressions known to be involved in the regulation of bone remodeling and mesenchymal stem cell commitment $(32,33,34,39)$. Cell culture research suggests that miR199a is a BMP-2-responsive miR, which inhibits early chondrogenesis through its direct effect on SMAD1 levels (27). In our research, both BMP2 and miR-199a-5p were downregulated. We suggest that the downregulation of miR-199a-5p could increase SMAD1 levels and to improve chondrocyte proliferation (38).

miR-29a, b and c were all downregulated in bone tissue samples of patients with acromegaly. miR29a modulates Wnt signaling in human osteoblasts through a positive feedback loop, as DKK1, KREMEN2 and secreted frizzled-related protein 2 are direct targets of miR-29a (18). In addition to this, the upregulation of miR-29a and miR-29 correlated with decreased osteonectin protein during the matrix maturation and mineralization phases (19).

miR-328-3p was more than twofold upregulated in bone tissue samples in the present study. This miR was decreased in the serum of patients with osteoporosis and suggested as a serum biomarker to reveal osteoporotic fracture and osteoporosis in postmenopausal women (32).

It is believed that GH affects the commitment of mesenchymal precursors, favoring osteoblastogenesis and chondrogenesis and opposing adipogenesis (1). However, it is unknown whether this is the case in acromegalic patients. Several of the tested miRs are essential for the shift from osteogenic to adipogenic differentiation of mesenchymal stem cells. miR-27a directly targets peroxisome proliferator-activated receptor gamma (PPARG) and gremlin 1 (GREM1) expression $(24,25,26)$. Consequently, the decreased levels of miR-27a-5p shown in this study might promote adipogenic differentiation of mesenchymal stem cells. miR-188-3p, which was upregulated in bone tissue samples of acromegalic patients, was also shown to be a key regulator of the switch between osteogenesis and adipogenesis of bone marrow mesenchymal stem cells. Upregulation of miR188-3p led to reduced bone formation and increased bone marrow fat accumulation in mice (22). miR-125b-5p, which was upregulated in the present research, has been shown to inhibit osteogenic differentiation through the regulation of cell proliferation and mesenchymal stem cell commitment $(20,21)$. We also found miR-34a-5p to be upregulated which, according to literature, may reduce the levels of cell cycle-related proteins (CDK4, CDK6, Cyclin D1) (23) leading to the inhibition of proliferation and therefore the inhibition of osteoblastogenesis.

Consequently, the downregulation of miR-27a-5p, miR29 a,b,c and upregulation of miR-125b-5p, miR188-3p, miR-34a-5p in bone tissue samples of patients with acromegaly is likely to contribute to the suppression of osteogenesis.

Other changes in miR profiles seem to be protective of bone formation. MmiR-204/211 act as endogenous negative regulators of RUNX2 (30) and both were downregulated in bone tissue samples of patients with acromegaly. miR-23a represses RUNX2 in the terminally differentiated osteocyte, representing a feedback mechanism to attenuate osteoblast maturation (31). miR-135, which was downregulated in patients with acromegaly, targets SMAD5, a key transducer of the BMP2 osteogenic signal (29). MiR-210, which was upregulated in this study, acts as a positive regulator of osteoblast differentiation and was shown to promote the BMP4-induced osteoblast differentiation of bone marrowderived ST2 stromal cells (28).

Interestingly, we found that miR-21-5p, but not miR-21-3p was upregulated in bone tissue samples from patients with acromegaly. miR-21-5p promotes 
osteogenic differentiation and impairs adipogenic differentiation through SPRY1 inhibition (40). However, it is also involved in the regulation of osteoclast activity and survival $(41,42)$.

Several potential functions have been reported for miR-31 (43, 44, 45), which was 50\% downregulated in bone tissue samples of patients with acromegaly. In human mesenchymal stem cells the overexpression of miR-31 repressed osteogenesis, whereas the downregulation of miR-31 enhanced it (43). In another report the overexpression of miR-31 significantly reduced the expression of osteogenic transcription factors, but not RUNX2 (44). In addition to this, the downregulation of miR-31-5p may attenuate RANKLinduced osteoclastogenesis and bone resorption through the upregulation of RHOA (45).

In the present study, we also discovered increased expression of miR-9-5p. According to current research $(46,47)$, miR-9 is involved in the inhibition of cell proliferation and survival in chondrogenic progenitors and articular chondrocytes by targeting protogenin (46). miR-9-5p was increased in cartilage samples from patients with osteoarthritis (47).

This is the first study documenting gene expression in bone tissue samples of patients with active acromegaly and has certain limitations. We used whole bone tissue samples that could have included bone marrow and we could not distinguish whether the induced transcriptional changes reflected changes in cellular composition or the response of a particular cell type. We also obtained bone tissue samples from the sphenoid bone (sella turcica) during neurosurgery, whereas usually a bone biopsy is taken from the iliac crest. Nevertheless, the part of the iliac crest ' $2 \mathrm{~cm}$ posterior to the anterior iliac crest' where the transiliac bone biopsy must be performed (48) is not weight-bearing, similar to the sphenoid bone. Classification of bone according to their geometrical characteristics includes vertebrae, pelvis and bones in the base of skull (specifically the sphenoid bone) in the same group - irregular bone (49). Considering the increased risk of vertebral fractures in patients with acromegaly, bone tissue samples from vertebrae would be ideal. At the same time, due to the systemic effects of IGF1 and $\mathrm{GH}$ on bone, similar outcomes in gene expression should be expected from different parts of the skeleton. In similar research on patients with Cushing's disease, bone tissue samples from the sphenoid bone showed downregulation of genes involved in osteoblast function and maturation (ACP5, ALPL, BGLAP, COL1A1, COL1A2, BMP2, RUNX2, TWIST1) (50), similar to a previous publication on gene expressions in lumbar vertebrae of glucocorticoid-treated mice (51).

Another possible limitation is the fact that both the patients with acromegaly and the control group had vitamin D deficiency and their gonadal function may be affected by the presence of pituitary tumor. However, there were no differences in vitamin $\mathrm{D}$ levels or hypogonadism between the groups and therefore the found differences in gene expressions were not related to vitamin D deficiency or gonadal status. Additionally, active D-hormone $(1,25(\mathrm{OH}) \mathrm{D} 3)$, which affects gene expression, is expected to be normal even in vitamin D deficient patients (52). Although we did not measure other metabolites of vitamin D or PTH values, calcium levels within the reference range and normal or slightly higher BMD values in all enrolled patients indicate that the condition is clinically compensated in both groups of patients. Nevertheless, we cannot exclude osteomalacia in both groups of patients, as we did not perform histological evaluation. We did not treat vitamin $\mathrm{D}$ deficiency before the enrollment because there are conflicting data on the effects of vitamin D supplementation on IGF1 in adults $(54,55)$ and it is known that IGF1 increases the circulating levels of 1,25-dihydroxyvitamin D by stimulating the expression and activity of $1 \alpha$-hydroxylase (54).

It is important to emphasize, that our findings outline the changes in the complex, long-term condition of acromegaly in humans rather than the effects of excess of GH or IGF1 on bone tissue.

We only evaluated the expression of genes which were previously reported to be associated with bone remodeling. However, we cannot be sure that we assessed all the relevant genes. In addition to this, it is impossible to provide a precise duration of acromegaly as it is frequently present for many years before it is diagnosed (53). This is a cross-sectional study with the consequent limitations related to the study design.

In summary, acromegaly has minimal effects on the tested mRNA specific to osteoblast or osteoclast function except for $A L P$ expression, which was 50\% downregulated in bone tissue samples. Consequently, in patients with acromegaly the increased bone remodeling markers in circulation do not reflect increased bone remodeling rate. We suggest that processes similar to endochondral ossification occur in patients with acromegaly and these explain the high levels of bone remodeling markers and normal or high BMD. The final stages of endochondral ossification require alkaline phosphatase and as this mRNA expression was downregulated and demonstrated 
strong negative association with IGF1, we suggest that it may alter the final stages of bone formation and bone quality.

Acromegaly affects osteoblastogenesis through a marked increase in the mRNA levels of both the Wnt signaling antagonist (DKK1) and agonist (WNT10B) and also through changes in the expressions of miR known to be involved in mesenchymal stem cell commitment. These findings along with downregulated TWIST1 expression, suggest predominant commitment to cartilage or adipocyte cells over mature osteoblasts.

In long-term acromegaly, these changes may lead to immaturity or deteriorated quality of newly formed bone with consequent increased bone fragility.

\section{Declaration of interest}

Zhanna Belaya, Tatiana Grebennikova, Galina Melnichenko, Alexey Nikitin, Alexander Solodovnikov, Olga Brovkina, Andrey Grigoriev, Liudmila Rozhinskaya, Lutsenko Alexander, Ivan Dedov declare that they have no conflict of interest.

Funding

Russian Scientific Foundation 15-15-30032

\section{Acknowledgments}

The authors thank Prof. Adrian Daly for valuable advice and Geoffrey Clayson for his English editing work.

\section{References}

1 Giustina A, Mazziotti G \& Canalis E. Growth hormone, insulin-like growth factors and the skeleton. Endocrine Reviews 200829 535-559. (https://doi.org/10.1210/er.2007-0036)

2 Claessen KM, Mazziotti G, Biermasz NR \& Giustina A. Bone and joint disorders in acromegaly. Neuroendocrinology 2016103 86-95. (https:// doi.org/10.1159/000375450)

3 Katznelson L, Laws ER, Melmed JS, Molitch ME, Murad MH, Utz A $\&$ Wass AH. Acromegaly: an endocrine society clinical practice guidelines. Journal Clinical Endocrinology and Metabolism 201499 3933-3951. (https://doi.org/10.1210/jc.2014-2700)

4 Hubsch P, Koltzmann H, Svoboda T, Kainberger FM, Bankier A \& Seidl G. Bone density of the lumbar spine and femur in acromegaly. Radiologe 199333 457-461.

5 Vestergaard P \& Mosekilde L. Fracture risk is decreased in acromegaly - a potential beneficial effect on growth hormone. Osteoporosis International 200415 155-159. (https://doi.org/10.1007/s00198-0031531-z)

6 Mazziotti G, Biagioli E, Maffezzoni F, Spinello M, Serra V, Maroldi R, Floriani I \& Giustina A. Bone turnover, bone mineral density, and fracture risk in acromegaly: a meta-analysis. Journal Clinical Endocrinology and Metabolism 2015100 384-394. (https://doi. org/10.1210/jc.2014-2937)

7 Silva PP, Ghazanfari Amlashi F, Yu EW, Pulaski-Liebert KJ, Gerweck A, Fazeli P, Lawson EA, Nachtigall LB, Biller BMK, Miller KK et al. Bone microarchitecture and estimated bone strength in men with active acromegaly. European Journal of Endocrinology 2017177 409-420. (https://doi.org/10.1530/EJE-17-0468)

8 Hong AR, Kim JH, Kim SW, Kim SY \& Shin CS. Trabecular bone score as a skeletal fragility index in acromegaly patients. Osteoporosis International 201627 1123-1129. (https://doi.org/10.1007/s00198015-3344-2)

9 Hofbauer LC, Hamann C \& Ebelling P. Approach to the patient with secondary osteoporosis. European Journal of Endocrinology 2010162 1009-1020. (https://doi.org/10.1530/EJE-10-0015)

10 Daly AF, Burlacu MC, Livadariu E \& Beckers A. The epidemiology and management of pituitary incidentalomas. Hormone Research 200768 195-198. (https://doi.org/10.1159/000110624)

11 Camacho PM, Petak SM, Binkley N, Clarke BL, Harris ST, Hurley DL, Kleerekoper M, Lewiecki EM, Miller PD, Narula HS et al. American association of clinical endocrinologists and American college of endocrinology clinical practice guidelines for the diagnosis and treatment of postmenopausal osteoporosis. Endocrine Practice 201622 1-42. (https://doi.org/10.4158/EP161435.GL)

12 Genant HK, Wu CY, van Kujik C \& Nevitt MC. Vertebral fracture assessment using a semiquantative technique. Journal of Bone and Mineral Research 19938 1137-1148. (https://doi.org/10.1002/ jbmr.5650080915)

13 Reiner A, Yekutieli D \& Benjamini Y. Identifying differently expressed genes using false discovery rate controlling procedures. Bioinformatics 200319 368-375. (https://doi.org/10.1093/bioinformatics/btf877)

14 Hirakawa A, Sato Y, Sozu T, Hamada C \& Yoshimura I. Estimating the false discovery rate using mixed normal distribution for identifying differentially expressed genes in microarray data analysis. Cancer Informatics 200822 140-148.

15 Baron R \& Kneissel M. Wnt signaling in bone homeostasis and disease: from human mutations to treatments. Nature Medicine 2013 19 179-192. (https://doi.org/10.1038/nm.3074)

16 Grebennikova TA, Belaya ZE, Rozhinskaya LY \& Melnichenko GA. The canonical Wnt/ $\beta$-catenin pathway: from the history of its discovery to clinical application. Terapevticheskii Arkhiv $2016 \mathbf{8 8}$ 74-81. (https://doi.org/10.17116/terarkh201688674-81)

17 Huang W, Yang S, Shao J \& Li Y. Signaling and transcriptional regulation in osteoblast commitment and differentiation. Frontiers in Bioscience 2013 12 3068-3092.

18 Kapinas K, Kessler C, Ricks T, Gronowicz G \& Delany AM. miR-29 modulates Wnt signaling in human osteoblasts through a positive feedback loop. Journal of Biological Chemistry $201028525221-25231$. (https://doi.org/10.1074/jbc.M110.116137)

19 Kapinas K, Kessler CB \& Delany AM. miR-29 suppression of osteonectin in osteoblasts: regulation during differentiation and by canonical Wnt signaling. Journal of Cellular Biochemistry 2009108 216-224. (https://doi.org/10.1002/jcb.22243)

20 Chen S, Yang L, Jie Q, Lin Y-S, Meng G-L, Fan J-Z, Zhang J-K, Fan J, Luo Z-J \& Liu J. MicroRNA-125b suppresses the proliferation and osteogenic differentiation of human bone morrow-derived mesenchymal stem cells. Molecular Medicine Reports 20149 1820-1826. (https://doi.org/10.3892/mmr.2014.2024)

21 Mizuno Y, Yagi K, Tokuzawa Y, Kanesaki-Yatsuka Y, Suda T, Katagiri T, Fukuda T, Maruyama M, Okuda A, Amemiya T et al. miR-125b inhibits osteoblastic differentiation by downregulation of cell proliferation. Biochemical and Biophysical Research Communications 2008368 267-272. (https://doi.org/10.1016/j.bbrc.2008.01.073)

22 Li CJ, Cheng P, Liang MK, Chen YS, Lu Q, Wang JY, Xia ZY, Zhou HD, Cao X, Xie H et al. MicroRNA-188 regulates age-related switch between osteoblast and adipocyte differentiation. Journal of Clinical Investigation 2015125 1509-1522. (https://doi.org/10.1172/ JCI77716)

23 Kang H, Chen H, Huang P, Qi J, Qian N, Deng L \& Guo L. Glucocorticoids impair bone formation of bone marrow stromal stem cells by reciprocally regulating microRNA-34a-5p. Osteoporosis 
International 201627 1493-1505. (https://doi.org/10.1007/s00198015-3381-x)

24 You L, Pan L, Chen L, Gu W \& Chen J. miR-27a is essential for the shift from osteogenic differentiation to adipogenic differentiation of mesenchymal stem cells in postmenopausal osteoporosis. Cellular Physiology and Biochemistry 201639 253-265. (https://doi. org/10.1159/000445621)

25 Gu C, Xu Y, Zhang S, Guan H, Song S, Wang X, Wang Y, Li Y $\&$ Zhao G. miR-27a attenuates adipogenesis and promotes osteogenesis in steroid-induced rat BMSCs by targeting PPAR $\gamma$ and GREM1. Scientific Reports 20166 38491. (https://doi.org/10.1038/ srep38491)

26 Guo D, Li Q, Lv Q, Wei Q, Cao S \& Gu J. miR-27a targets sFRP1 in hFOB1 cells to regulate proliferation, apoptosis and differentiation. PLoS ONE 20149 e91354. (https://doi.org/10.1371/journal. pone.0091354)

27 Lin EA, Kong L, Bai XH, Luan Y \& Liu CJ. miR-199a, a bone morphogenic protein 2-responsive microRNA regulates chondrogenesis via direct targeting to Smad1. Journal of Biological Chemistry 2009284 11326-11335. (https://doi.org/10.1074/jbc. M807709200)

28 Mizuno Y, Tokuzawa Y, Ninomiya Y, Yagi K, Yatsuka-Kanesaki Y, Suda T, Fukuda T, Katagiri T, Kondoh Y, Amemiya T et al. miR-210 promotes osteoblastic differentiation through inhibition of AcvR1b. FEBS Letters 200920583 2263-2268. (https://doi.org/10.1016/j. febslet.2009.06.006)

29 Li Z, Hassan MQ, Volinia S, van Wijnen AJ, Stein JL, Croce CM, Lian JB \& Stein GS. A microRNA signature for a BMP2-induced osteoblast lineage commitment program. PNAS 2008105 13906-13911. (https://doi.org/10.1073/pnas.0804438105)

30 Huang J, Zhao L, Xing L \& Chen D. MicroRNA-204 regulates Runx2 protein expression and mesenchymal progenitor cell differentiation. Stem Cells 201028 357-364.

31 Hassan MQ, Gordon JA, Beloti MM, Croce CM, van Wijnen AJ, Stein JL, Stein GS \& Lian JB. A network connecting Runx2, SATB2, and the miR--23a 27a 24-2 cluster regulates the osteoblast differentiation program. PNAS 2010107 19879-19884. (https://doi. org/10.1073/pnas.1007698107)

32 Weilner S, Skalicky S, Salzer B, Keider V, Wagner M, Hildner F, Gabriel C, Dovjak P, Pietschmann P, Grillari-Voglauer R et al. Differentially circulating miRNAs after recent osteoporotic fractures can influence osteogenic differentiation. Bone 201579 43-51. (https://doi.org/10.1016/j.bone.2015.05.027)

33 Lian JB, Stein GS, van Wijnen AJ, Stein JL, Hassan MQ, Gaur T \& Zhang Y. MicroRNA control of bone formation and homeostasis. Nature Reviews Endocrinology 20128 212-227. (https://doi. org/10.1038/nrendo.2011.234)

$34 \mathrm{Ji}$ X, Chen X \& Yu X. MicroRNAs in osteoclastogenesis and function: potential therapeutic targets for osteoporosis. International Journal of Molecular Sciences 201617 349. (https://doi.org/10.3390/ ijms17030349)

35 Weiner JA \& Jontes JD. Protocadherins, not prototypical: a complex tale of their interactions, expression, and functions. Frontiers in Molecular Neuroscience 201364.

36 Kosa JP, Balla B, Speer G, Kiss J, Borsy A, Podani J, Takacs I, Lozary A, Nagy Z, Bacsi K et al. Effect of menopause on gene expression pattern in bone tissue of nonosteoporotic women. Menopause 200916 367-377. (https://doi.org/10.1097/gme.0b013e318188b260)

37 Fogelman J, Gnanasegaran G \& van der Wall H Eds. Radionuclide and Hybrid Bone Imaging. Berlin, Heidelberg: Springer-Verlag, 2012, 29. (https://doi.org/10.1007/978-3-642-02400-9_2)

38 Retting KN, Song B, Yoon BS \& Lyons KM. BMP canonical Smad signaling through Smad1 and Smad5 is required for endochondral bone formation. Development 2009136 1093-1104. (https://doi. org/10.1242/dev.029926)
39 Jing D, Hao J, Shen Y, Tang G, Li M, Huang S \& Zhao Z. The role of microRNA in bone remodeling. International Journal of Oral Science 20157 131-143. (https://doi.org/10.1038/ijos.2015.22)

40 Trohatou O, Zagoura D, Bitsika V, Pappa KI, Antsakis A, Anagnou NP \& Roubelakis MG. Sox2 suppression by miR-21 governs human mesenchymal stem cells properties. Stem Cells Translational Medicine 20143 54-68. (https://doi.org/10.5966/ sctm.2013-0081)

41 Sugatani T \& Hruska KA. Downregulation of miR-21 biogenesis by estrogen action contributes to osteoclastic apoptosis. Journal of Cellular Biochemistry 2013114 1217-1222. (https://doi.org/10.1002/ jcb.24471)

42 Yang N, Wang G, Hu C, Shi Y, Liao L, Shi S, Cai Y, Cheng S, Wang X $\&$ Liu Y. Tumor necrosis factor $\alpha$ suppresses the mesenchymal stem cell osteogenesis promote miR-21 in estrogen deficiency-induced osteoporosis. JBMR 201328 559-573. (https://doi.org/10.1002/ jbmr.1798)

43 Xie Q, Wang Z, Bi X, Zhou H, Wang Y, Gu P \& Fan X. Effects of miR-31 on the osteogenesis of human mesenchymal stem cells. Biochemical and Biophysical Research Communications 2014446 98-104. (https://doi.org/10.1016/j.bbrc.2014.02.058)

44 Deng Y, Wu S, Zhou H, Wang Y, Hu Y, Gu P \& Fan X. Effects of a miR-31, Runx2, and Satb2 regulatory loop on the osteogenic differentiation of bone mesenchymal stem cells. Stem Cells and Development 201322 2278-2286. (https://doi.org/10.1089/ scd.2012.0686)

45 Mizoguchi F, Murakami Y, Saito T, Miyasaka N \& Kohsaka H. miR31 controls osteoclast formation and bone resorption by targeting RhoA. Arthritis Research and Therapy 201315 R102. (https://doi. org/10.1186/ar4282)

46 Song J, Kim D, Chun C-H \& Jin E-J. MicroRNA-9 regulates survival of chondroblasts and cartilage integrity by targeting protogenin. Cell Communication and Signaling 201311 11-66. (https://doi. org/10.1186/1478-811X-11-11)

47 Gu R, Liu N, Luo S, Huang W, Zha Z \& Yang J. MicroRNA-9 regulates the development of knee osteoarthritis through the NF-kappab1 pathway in chondrocytes. Medicine 201695 e4315. (https://doi. org/10.1097/MD.0000000000004315)

48 Hernandez JD, Wesseling K, Pereira R, Gales B, Harrison R \& Salusky IB. Technical approach to iliac crest biopsy. Clinical Journal of the American Society of Nephrology 20083 (Supplement 3) S164-S169. (https://doi.org/10.2215/CJN.00460107)

49 Isaza SJ. Characterization of the mechanical and morphological properties of cortical bones by nanoindentation and Atomic Force Microscopy. Biomechanics [physics.med- ph]. Université de Technologie de Compiégne 2014. English. ¡ NNT: 2014COMP2150.

50 Belaya ZE, Grebennikova TA, Melnichenko GA, Nikitin AG, Solodovnikov AG, Brovkina OI, Grigoriev AV, Rozhinskaya LY $\&$ Dedov II. Effects of endogenous hypercortisolism on bone mRNA and microRNA expression in humans. Osteoporosis International 201829 211-221. (https://doi.org/10.1007/s00198. 017-4241-7)

51 Yao W, Cheng Z, Busse C, Pham A, Nakamura MC \& Lane NE. Glucocorticoid excess in mice results in early activation of osteoclastogenesis and prolonged suppression of osteogenesis. Arthritis and Rheumatology 200858 1674-1686. (https://doi. org/10.1002/art.23454)

52 Holick MF. Vitamin D status: measurement, interpretation and clinical application. Annals of Epidemiology 200919 73-78. (https:// doi.org/10.1016/j.annepidem.2007.12.001)

53 Petrossians P, Daly AF, Natchev E, Maione L, Blijdorp K, SahnounFathallah M, Auriemma R, Diallo AM, Hulting AL, Ferone D et al. Acromegaly at diagnosis in 3173 patients from the Liège Acromegaly Survey (LAS) Database. Endocrine-Related Cancer 201724 505-518. (https://doi.org/10.1530/ERC-17-0253) 
54 Ameri P, Giusti A, Boschetti M, Bovio M, Teti C, Leoncini G, Ferone D, Murialdo G \& Minuto F. Vitamin D increases circulating IGF1 in adults: potential implication for the treatment of GH deficiency. European Journal of Endocrinology 2013169 767-772. (https://doi.org/10.1530/EJE-13-0510)
55 Trummer C, Schwetz V, Pandis M, Grubler MR, Verheyen N, Gaksch M, Zittermann A, Marz W, Aberer F, Lang A et al. Effects of Vitamin D supplementation on IGF-1 and calcitriol: a randomizedcontrolled trial. Nutrients 20179 623. (https://doi.org/10.3390/ nu9060623)

Received 19 September 2017

Revised version received 5 January 2018

Accepted 26 January 2018 